Columbia International Publishing

Journal of Continuing Education and Professional Development

(2015) Vol. 2 No. 1 pp. 1-8

doi:10.7726/jcepd.2015.1001

Research Article

\title{
Increasing Capacity in Rehabilitation in the Management of HIV: A Case-based Email Intervention
}

\author{
Patricia Solomon, $\mathrm{PhD}^{1 *}$, Nancy Salbach, $\mathrm{PhD}^{2}$, Kelly K. O’Brien, $\mathrm{PhD}{ }^{2}$, Cathy Worthington, $\mathrm{PhD}^{3}$, \\ Larry Baxter, $\mathrm{BA}^{4}$, Georgina Blanchard, $\mathrm{MA}^{4}$, Alan Casey, MD, FRCPC ${ }^{5}$, Will Chegwidden, \\ BSc(OT)(Hons) ${ }^{6}$, Le-Ann Dolan, BSW ${ }^{4}$, Sarah Eby, Hons BSc (Biomedical sciences), $\mathrm{MScPT}^{2}$, and \\ Nicole Gervais, MA $^{1}$
}

Received 25 July 2014; Published online 22 November 2014

(C) The author(s) 2014. Published with open access at www.uscip.us

\begin{abstract}
Despite evidence of the role of rehabilitation in the management of HIV related disability, few rehabilitation professionals work in HIV care. Barriers include a dearth of knowledge about potential roles of rehabilitation professionals and an absence of research evidence. This study evaluated the extent to which case-based emails with links to an online guide to HIV rehabilitation increased awareness and capacity in rehabilitation professionals. 26 rehabilitation professionals were emailed 6 case studies highlighting salient issues related to HIV and rehabilitation and directing participants to the guide. Following the intervention, each participant completed an interview to assess uptake and impact of the guide. The interviews underwent a qualitative content analysis. Participants increased or reinforced their knowledge, increased confidence in the management of HIV, and recognized the relevance of prior knowledge and skills to HIV care. Clinical cases integrated as an email intervention can promote learning and use of an electronic evidence-informed resource.
\end{abstract}

Keywords: Rehabilitation; HIV; Disability; Case Studies; Learning Module; Capacity Building

\section{Introduction}

The availability of combination antiretroviral therapy has transformed HIV from a palliative to chronic illness. People living longer with HIV often experience disability in the form of symptoms and impairments, difficulties with day-to-day activities, challenges to social inclusion, and

*Corresponding e-mail: solomon@mcmaster.ca

$1^{*}$ McMaster University, Hamilton, ON, Canada

2 University of Toronto, Toronto, ON, Canada

3 University of Victoria, Victoria, BC, Canada

4 Canadian Working Group on HIV and Rehabilitation, Toronto, ON, Canada

5 Health Sciences Centre Winnipeg, Winnipeg, MB, Canada

6 University College Hospitals NHS Foundation Trust, London, England, United Kingdom 


\section{Patricia Solomon, Nancy Salbach, Kelly K. O'Brien, Cathy Worthington, Larry Baxter, Georgina Blanchard, Alan Casey, Will Chegwidden, Le-Ann Dolan, Sarah Eby, and Nicole Gervais / Journal of Continuing Education and Professional Development (2015) Vol. 2 No. 1 pp. 1-8}

uncertainty (O'Brien et al., 2008). Rehabilitation may be broadly defined as interventions that address physical, emotional, social and professional challenges, and has an important role in managing disability and increasing quality of life for People living with HIV/AIDS (PHAs; Worthington et al., 2005). Despite increasing evidence of the role of rehabilitation in the management of PHAs (e.g., O'Brien et al., 2010) few rehabilitation professionals work in HIV care (Worthington et al., 2008).

Health professionals often struggle to keep current with research literature and best practices (Grimshaw et al., 2012). This challenge is accentuated in the emerging field of HIV and rehabilitation in which there is a dearth of knowledge about potential roles of rehabilitation professionals and an absence of synthesized research evidence. Recognizing the need to bridge the gap between knowledge and practice, the Canadian Working Group on HIV and Rehabilitation (CWGHR) developed a comprehensive evidence-informed online guide to HIV rehabilitation (emodule; CWGHR 2011). CWGHR's intent was to provide accessible information about HIV tailored to rehabilitation professionals such as occupational therapists (OTs), physiotherapists (PTs) and speech-language pathologists (SLPs).

Integrating research findings with clinical expertise, and patient preferences and values, is the goal of evidence-informed rehabilitation (MacDermid et al., 2006). There is a paucity of evidence on the effectiveness of knowledge translation interventions among rehabilitation professionals (Menon et al., 2009). However, systematic reviews of interventions aimed at changing health professional behavior have shown a variety of interventions including the dissemination of educational materials to have modest effects (Grimshaw et al., 2004). Case studies sent by e-mail have been used as an effective means of continuing health professional education, resulting in significant improvements in knowledge, confidence and communication compared to controls (Kemper et al., 2002). We were interested in promoting awareness and use of the e-module, available as a .pdf file online, by engaging rehabilitation professionals in a series of emailed case studies related to HIV that contained links to salient components of the e-module.

The objective of this study was to evaluate the extent to which case-based emails with content and links to the e-module increased awareness and capacity in rehabilitation professionals to provide evidence-informed healthcare for people living with HIV.

\section{Methods}

\subsection{The E-Module for Evidence-Informed HIV Rehabilitation}

The e-module is an openly accessible comprehensive resource designed to enhance knowledge about HIV care among rehabilitation professionals and enable them to better address the needs of PHAs (CWGHR, 2011). The e-module incorporates current best evidence and promotes an interprofessional approach to rehabilitation management. The 223 page e-module is comprised of eight chapters including an introduction, overview of best practices in rehabilitation, symptoms and impairments, systemic impacts, pediatrics, interventions and rehabilitation related issues. A final chapter includes six case studies to encourage integration of knowledge. 


\section{Patricia Solomon, Nancy Salbach, Kelly K. O'Brien, Cathy Worthington, Larry Baxter, Georgina Blanchard, Alan Casey, Will Chegwidden, Le-Ann Dolan, Sarah Eby, and Nicole Gervais / Journal of Continuing Education and Professional Development (2015) Vol. 2 No. 1 pp. 1-8}

We conducted a single-group intervention study using in-depth telephone interviews to assess the uptake and impact of the e-module among rehabilitation professionals. Eligible participants included OTs, PTs or SLPs who were registered with their professional regulatory body and provided direct clinical care. Rehabilitation professionals in Canada and the United Kingdom (UK) were recruited through emails to members of the CWGHR, the Rehabilitation and HIV Association (RHIVA), professional associations and special interest groups. Participants completed a questionnaire at baseline to determine socio-demographic and clinical practice characteristics. The intervention consisted of six clinical case studies adapted from those in the e-module to highlight current knowledge on the pathophysiology of HIV and associated conditions, assessment and treatment of HIV, and the complexities of living with HIV. The case studies were sent to participants in the body of an email. Embedded within the case studies were key clinical questions applying rehabilitation guidelines to PHAs along with answers and links to specific content in the emodule to encourage use of this resource. We sent a case-study email every two weeks for a three month period for a total of six case studies. A certificate of completion was provided to all participants.

Following the three month intervention, participants completed an in-depth telephone interview to assess their perceptions of the value of the case studies, their use of the e-module and barriers and facilitators to using the e-module. Interviews were audio-recorded and transcribed verbatim.

Study approval was received from the Research Ethics Boards of McMaster University, University of Toronto and University of Victoria.

\subsection{Analysis}

Interview data were analyzed using qualitative content analysis and an open coding technique (Hsieh and Shannon, 2005). Three investigators independently read 4 transcripts to develop a preliminary coding framework. The investigators then coded the remaining transcripts independently meeting periodically to compare codes, reconcile differences and revise the coding scheme. The investigators then met to review the codes and transcripts to identify broader categories and salient quotes.

\section{Results}

Participants were recruited through advertisements with CWGHR, RHIVA in the UK, and various provincial and national professional associations for PT, OT and SLP in Canada. Thirty eligible rehabilitation professionals volunteered to participate; 26 viewed all 6 case-study emails and completed the study tasks (13 Canadian PTs, 4 Canadian OTs, 3 each of PT, OT and SLP from the UK). Four participants began the study but were unable to continue due to time restrictions. Two rehabilitation professionals expressed interest but were ineligible for the study. Of the 26 participants, 96\% were female with a mean age of 34 years (range 23-48 years) and a mean 8.3 years of clinical experience (range 0-25 years). Nineteen percent ( 4 from the UK and 1 from Canada) worked 'extensively' with PHAs (defined as seeing more than 10 HIV positive patients a year; while 35\% ( 1 from the UK and 8 from Canada) had never knowingly treated anyone living with HIV. Clinicians practiced in a wide variety of settings: $11(42 \%)$ in acute care, 4 (15\%) in a 


\section{Patricia Solomon, Nancy Salbach, Kelly K. O'Brien, Cathy Worthington, Larry Baxter, Georgina Blanchard, Alan Casey, Will Chegwidden, Le-Ann Dolan, Sarah Eby, and Nicole Gervais / Journal of Continuing Education and Professional Development (2015) Vol. 2 No. 1 pp. 1-8}

rehabilitation hospital, 3 (12\%) in private practice, and 1 each (4\%) in a general hospital, primary health care, community health centre, home visiting agency, day care and schools. One participant was not currently practicing. Analyses revealed two broad themes of 1 ) increased or reinforced knowledge and 2) increased confidence about HIV rehabilitation. These contained 7 subthemes as described below with representative quotes.

\subsection{Increased or Reinforced Knowledge}

Participants described broad areas in which they perceived an increase in knowledge as a result of the e-mail intervention. This was true for therapists from a variety of levels of experience, though those who had worked previously with PHAs articulated that their knowledge was reinforced or updated.

Multi-morbidities or co-morbidities: Participants described specific factual knowledge gained through the cases (e.g., pathophysiology, associated conditions, effects of antiretroviral therapy), particularly among those with little prior knowledge of HIV. The fact that many living with HIV experience complexities related to multi-morbidities or co-morbidities was a new area of learning for those with little prior knowledge of HIV.

(Participating in the project has) changed my ideas about what kind of questions I need to ask and how I can be more helpful in adjusting some of the needs (of people living with HIV). Especially what I said before about some of the co-morbidities that I didn't really know, just so that I have more of an idea of what I'm seeing, how I can educate for signs to look for, or things that (PHAs) have to be mindful of especially because it's episodic. I feel more equipped especially on the education aspect for when I am interacting with people.

Physiotherapist (Canada)

Potential for rehabilitation: Participants gained a greater appreciation of the potential of rehabilitation for PHAs. They described how the case studies made them realize that a diversity of healthcare professionals have a role in HIV rehabilitation. Some viewed HIV as a terminal and palliative illness and were surprised at the potential scope of rehabilitation and the number of health and social service providers that could be engaged.

I think I will be more involved than I might have been in the past. So knowing that (PHAs have) rehab[ilitation] potential and that we've got a really strong role for rehabilitating them. I'll try and be a little bit less medical model and a little bit more social focus ....like getting them into their home environment if it's appropriate.

Speech Language Therapist (UK)

Many had not associated HIV with disability and this revelation helped them to better envision a role for their profession.

I didn't really appreciate that you could live in the community with HIV and be quite well and, this idea of (HIV) being a disability. 


\section{Patricia Solomon, Nancy Salbach, Kelly K. O'Brien, Cathy Worthington, Larry Baxter, Georgina Blanchard, Alan Casey, Will Chegwidden, Le-Ann Dolan, Sarah Eby, and Nicole Gervais / Journal of Continuing Education and Professional Development (2015) Vol. 2 No. 1 pp. 1-8}

Need for a holistic approach: Participants described an increased awareness of the complexities of living with HIV and the need for a holistic approach in their management. They described a greater appreciation of the diverse physical, cognitive and neurological HIV-related issues, as well as social, emotional, and psychological aspects of HIV that impact health-related quality of life. As a result, participants recognized their potential to have a role related to overall client/patient advocacy.

The e-module and the case studies did help me look more at the social complexities of this population as opposed to the clinical.

Speech Language Therapist (UK)

Episodic nature of HIV: The increased awareness of disability associated with the consequences of HIV was accompanied by the recognition of the episodic nature of HIV. Participants recognized how the episodic nature of HIV adds to its complexity and often requires greater use of health care services. They identified the episodic nature of HIV as a feature that distinguishes HIV from other chronic conditions, yet recognized similarities with other illnesses.

(People living with HIV) have periods where (the symptoms) are exacerbating and then they are fine ... that's similar to MS and other neurological ones. It gave me that perspective of those clients as well. It provides them with treatment that's appropriate when they are having good periods and bad periods and so it kind of helped to remind me about that.

Physiotherapist (Canada)

Importance of the team: Understanding the complexities of living with HIV highlighted the need for an interprofessional approach to care and the need to understand appropriate referral resources.

You need the reminder saying there is more than just the physio(therapy) aspect of it. And if you are the primary care provider that's the first thing, you need to know what's available, where Social Work comes in, where Occupational Therapy comes in, where Psychology comes in, where medical comes in.

Physiotherapist (Canada)

Although the target population was rehabilitation professionals, participants suggested that this information would be useful to other health professionals in order to increase awareness of the potential role of rehabilitation in all team members.

\subsection{Increased Confidence}

Participants, particularly those with little experience in managing HIV, described gaining a sense of increased confidence in the management of HIV as a consequence of learning through the case studies. Some of the confidence came through increased knowledge; other contributors are presented below.

Access to a comprehensive resource: Participants acknowledged that the e-module was a valuable resource for future reference. They described the challenges of searching for information and the convenience of having an easily accessible resource. Many recognized that if they were not 


\section{Patricia Solomon, Nancy Salbach, Kelly K. O'Brien, Cathy Worthington, Larry Baxter, Georgina Blanchard, Alan Casey, Will Chegwidden, Le-Ann Dolan, Sarah Eby, and Nicole Gervais / Journal of Continuing Education and Professional Development (2015) Vol. 2 No. 1 pp. 1-8}

encountering PHAs on a regular basis they would need to update their learning. Others appreciated knowing there was an extensive reference list to consult when they encountered unfamiliar issues or had clinical questions.

And the thing that I like now is that I do have something to refer to if I did have a patient come in who had HIV so I wouldn't feel lost and having to go search for these things on my own.

Physiotherapist (Canada)

Relevance of pre-existing knowledge and skills: Participants described a sense of confidence through recognition that there are similarities in assessment and management approaches between HIV and other chronic and episodic illnesses. Participants realized that their prior knowledge of common co-morbidities experienced by PHAs was applicable and foundational to management.

(HIV) has an element of unpredictability and episodic-ness to it and I'm thinking actually it's common to a number of other conditions which I feel really, really confident, you know, working on.

Occupational Therapist (UK)

\section{Discussion}

Our intent was to increase awareness and uptake of an evidence-informed guide to rehabilitation practice among rehabilitation professionals via a novel technique of dissemination. Findings suggest that providing an email knowledge translation intervention centered on case studies with embedded links to a comprehensive evidence-informed guide was useful in increasing knowledge of, and confidence in, applying rehabilitation strategies to PHAs. There was a high degree of uptake of the intervention as indicated by the percentage of participants who completed all 6 weeks of the intervention.

From a knowledge translation perspective, this intervention could be considered as a step related to selecting and implementing tailored interventions to promote guideline use (Graham et al., 2006). Our goals were modest; we aimed to increase awareness of the role of rehabilitation in the management and treatment of HIV and to facilitate use of the e-module. While we achieved our goal, the knowledge to action cycle is complex involving many more steps in moving evidence to practice (Graham et al., 2006).

Increased confidence among participants was an important consequence of the intervention. Many recognized that their pre-existing skills and knowledge were directly applicable to providing rehabilitation for PHAs. Not unexpectedly, as these are emergent areas of practice, rehabilitation professionals identified understanding the co-morbidities and episodic nature of illness experienced by those living with HIV as perceived areas of learning. However learning in areas more behaviorally science focused such as the need for a holistic and interprofessional approach to care were also important. PHAs may represent vulnerable populations hence, strategies for dealing with issues such as stigma and poverty should be integrated into overall management. An interprofessional team approach is an important element aspect of providing comprehensive care (Solomon et al., 2003). 


\section{Patricia Solomon, Nancy Salbach, Kelly K. O'Brien, Cathy Worthington, Larry Baxter, Georgina Blanchard, Alan Casey, Will Chegwidden, Le-Ann Dolan, Sarah Eby, and Nicole Gervais / Journal of Continuing Education and Professional Development (2015) Vol. 2 No. 1 pp. 1-8}

Case-based and problem-based learning are increasingly popular pedagogies in health professional education. Through providing a relevant context for learning, these pedagogies make learning more meaningful and effective (Solomon, 2005). Our cases were intended to engage learners with realistic scenarios to which they could apply current knowledge, recognize gaps in learning and be self-directed in their use of an electronically accessible module. To our knowledge this process has not been evaluated as a knowledge translation strategy and needs to be examined in other content areas.

While the e-module is not a systematically developed clinical practice guideline, it does attempt to synthesize current best practices relevant to HIV and rehabilitation. An important element to promoting implementation of CPGs, that of "usability," which relates to the ease with which guidelines can be used (Gagliardi and Brouwers, 2012), is applicable to our study. Through providing links to relevant content areas, participants can immediately access information that is most relevant to their learning thus enhancing usability.

One limitation to this study relates to the participants' varied knowledge of HIV and rehabilitation; some were directly involved in management of PHAs while others had no experience. These diverse learning needs may promote different use of the emails and e-module which we did not anticipate and evaluate. Another limitation relates to the representativeness of our participants. We advertised broadly through both HIV specific organizations, and more generally, to profession specific organizations. While only $19 \%$ of the participants indicated they worked extensively with PHAs, it is possible that those who volunteered represented a highly motivated group which contributed to the positive outcomes of this research.

This study provides evidence that clinical cases integrated as a multi-phased email intervention can promote learning and use of an electronic evidence-informed resource. Further research to assess whether this type of intervention can lead to changes in practice is warranted.

\section{Acknowledgements}

This research was funded by grant KAL-114093 from the Canadian Institutes of Health Research. Kelly K. O’Brien is supported by a Canadian Institutes of Health Research (CIHR) New Investigator Award.

\section{References}

Canadian Working Group on HIV and Rehabilitation (CWGHR). (2011). E-module for evidence-informed HIV rehabilitation (e-module). Toronto: Canadian Working Group on HIV and Rehabilitation.

Gagliardi, A. R., \& Brouwers, M. C. (2012). Integrating guideline development and implementation: analysis of guideline development manual instructions for generating implementation advice. Implementation Science, 7(67), 67. http://dx.doi.org/10.1186/1748-5908-7-67

Graham, I. D., Logan, J., Harrison, M. B., Straus, S. E., Tetroe, J., Caswell, W., \& Robinson, N. (2006). Lost in knowledge translation: time for a map? Journal of Continuing Education in the Health Professions, 26(1), 
Patricia Solomon, Nancy Salbach, Kelly K. O'Brien, Cathy Worthington, Larry Baxter, Georgina Blanchard, Alan Casey, Will Chegwidden, Le-Ann Dolan, Sarah Eby, and Nicole Gervais / Journal of Continuing Education and Professional Development (2015) Vol. 2 No. 1 pp. 1-8

13-24. doi: $10.1002 / \operatorname{chp} .47$

http://dx.doi.org/10.1002/chp.47

Grimshaw, J., Thomas, R., MacLennan, G., Fraser, C., Ramsay, C. R., Vale, L., ... \& Donaldson, C. (2004). Effectiveness and efficiency of guideline dissemination and implementation strategies. Health Technology Assessment, 8(6). http://dx.doi.org/10.3310/hta8060

Grimshaw, J. M., Eccles, M. P., Lavis, J. N., Hill, S. J., \& Squires, J. E. (2012). Knowledge translation of research findings. Implementation Science, 7(1), 50. http://dx.doi.org/10.1186/1748-5908-7-50

Hsieh H.F., Shannon S. E. (2005). Three approaches to qualitative content analysis. Qualitative Health Research, 15 (9), 1277-88. http://dx.doi.org/10.1177/1049732305276687

Kemper, K. J., Amata-Kynvi, A., Sanghavi, D., Whelan, J. S., Dvorkin, L., Woolf, A., . . Hibberd, P. (2002). Randomized Trial of an Internet Curriculum on Herbs and Other Dietary Supplements for Health Care Professionals. Academic Medicine, 77(9), 882-889. http://dx.doi.org/10.1097/00001888-200209000-00014

MacDermid, J.C., Solomon, P., Law, M., Russell, D., \& Stratford, P. (2006). Defining the effect and mediators of two knowledge translation strategies designed to alter knowledge, intent and clinical utilization of rehabilitation outcome measures: a study protocol [NCT00298727]. Implementation Science, 1, 14. http://dx.doi.org/10.1186/1748-5908-1-14

Menon, A., Korner-Bitensky, N., Kastner, M., McKibbon, K.A., \& Straus, S. (2009). Strategies for rehabilitation professionals to move evidence-based knowledge into practice: a systematic review. Journal of Rehabilitation Medicine, 41(13), 1024-1032. http://dx.doi.org/10.2340/16501977-0451

O'Brien, K., Bayoumi, A., Strike, C., Young, N., \& Davis, A. (2008). Exploring disability from the perspective of adults living with HIV/AIDS: development of a conceptual framework. Health and Quality of Life Outcomes, 6(1), 76. http://dx.doi.org/10.1186/1477-7525-6-76

O'Brien, K., Nixon, S., Tynan, A.M., \& Glazier, R. (2010). Aerobic exercise interventions for adults living with HIV/AIDS. The Cochrane Database of Systematic Reviews, 8, CD001796. http://dx.doi.org/10.1002/14651858.CD001796.pub3

Solomon, P. (2005). Problem-based learning: A review of current issues relevant to physiotherapy education. Physiotherapy theory and practice, 21(1), 37-49. http://dx.doi.org/10.1080/09593980590911499

Solomon P., Salvatori P, Guenter D. (2003). An Interprofessional Problem-based Learning Course on Rehabilitation Issues in HIV. Medical Teacher, 25(4), 408-413. http://dx.doi.org/10.1080/0142159031000137418

Worthington, C., Myers, T., O'Brien, K., Nixon, S., Cockerill, R., \& Bereket, T. (2008). Rehabilitation professionals and human immunodeficiency virus care: results of a national Canadian survey. Archives of Physical Medicine and Rehabilitation, 89(1), 105-113. http://dx.doi.org/10.1016/j.apmr.2007.10.009

Worthington C, Myers T, O'Brien K, Nixon S, \& Cockerill R. (2005). Rehabilitation and HIV/AIDS: Development of an expanded conceptual framework. AIDS Patient Care and STDs, 19(4), 258-71. http://dx.doi.org/10.1089/apc.2005.19.258 